\title{
Omphalocele and gastroschisis: An 18-year review study
}

Pei-Jen Hwang, MD, and Boris G. Kousseff, MD

\begin{abstract}
Purpose: Using data from a single University Genetics Division practice, the authors sought to determine if gastroschisis is increasing in occurrence compared to omphalocele. Associated abnormalities were also examined. In addition, prenatal exposure to teratogens and other parameters were compared. Methods: All fetal and infant cases of omphalocele and gastroschisis seen in the University of South Florida Division of Genetics between January 2, 1982 and December 31, 1999 were retrieved through the database and analyzed through chart review. Results: There were 127 cases of omphalocele and 121 cases of gastroschisis. Ninety-three of the 127 probands with omphalocele had karyotype determinations and $20 \%$ were abnormal; for gastroschisis, 37 had cytogenetic studies and none had chromosomal anomalies. Seventy-six percent of the probands with omphalocele had associated abnormalities; twenty-three percent of the probands with gastroschisis, none of which were syndromic, had associated anomalies. The prematurity rate for omphalocele was $42 \%$ and mortality was $22 \%$; for gastroschisis, the figures were $57 \%$ and $9 \%$, respectively. There were no significant differences in teratogen exposure between the two groups. Familial cases of both defects occurred in $<4 \%$, and the prevalence of dizygous twinning was increased in both groups. Mean maternal age was 21.8 years for the gastroschisis group, 27.2 years for the omphalocele group. Conclusions: In this study, the number of cases of omphalocele and gastroschisis were similar, compared to the expected 3:2 ratio, suggesting an increase in the occurrence of gastroschisis. Cases with omphalocele had more syndromic and nonsyndromic anomalies, more chromosomal anomalies, a higher mortality rate, and older mothers. Genet Med 2004:6(4):232-236.
\end{abstract}

Key Words: gastroschisis, omphalocele, abnormalities, teratogens

Gastroschisis and omphalocele are abdominal wall defects that were first described as early as the 16 th century. ${ }^{1}$ However, it was not until recently that these two conditions were categorized as separate entities. In 1953, Moore and Stokes ${ }^{2}$ defined the two separate conditions, and in 1963, Duhamel ${ }^{3}$ emphasized their distinct pathogenesis and clinical presentations. It is currently taught that omphalocele is a more common condition than gastroschisis, occurring in 1 per 4000 live births compared to 1 per 6000 for gastroschisis. ${ }^{4}$ Gastroschisis tends to be an isolated anomaly, whereas omphaloceles are frequently associated with chromosome abnormalities and other birth defects. ${ }^{5}$ Thus, the long-term prognosis for infants with gastroschisis is considerably better than that for infants with omphalocele, in whom a $50 \%$ to $60 \%$ survival rate and frequently chronic medical problems are seen. ${ }^{5}$

Using the records of a single University Division of Genetics over a period of 18 years, the occurrence of omphalocele and gastroschisis were compared, and associated abnormalities and karyotypes were contrasted in addition to maternal demo-

\footnotetext{
From the Division of Genetics, Department of Pediatrics, University of South Florida College of Medicine, Tampa, Florida.

Boris G. Kousseff, One Davis Blvd, Suite 604, Tampa, FL 33606.
}

DOI: 10.1097/01.GIM.0000133919.68912.A3 graphic features and outcomes of patients with these two conditions.

\section{MATERIALS AND METHODS}

A historical analysis of all probands with omphalocele and gastroschisis evaluated in the Genetics Division at the University of South Florida College of Medicine between January 2, 1982 and December 31, 1999 was performed. All cases were seen through the prenatal genetic clinics or as pediatric consultations, and were part of the 36,665 families evaluated by USF Genetics during the period. USF Genetics receives all genetic consultations requested by pediatricians and obstetricians within the Tampa Bay area counties. Probands were retrieved through the USF Genetics database and a chart analysis was performed on each proband. There were 127 probands with omphalocele (74 prenatal, 53 pediatric) and 121 with gastroschisis ( 92 prenatal, 29 pediatric).

For each record, karyotype, associated anomalies, prematurity, 1-year mortality rate, environmental exposures during pregnancy, maternal diabetes, familial recurrence, and maternal age were analyzed. Additional analyzed factors were incorrect or missed prenatal diagnosis, twinning, and race. In order to compare the two groups, $P$-values were calculated through the chi-square test. Polyhydramnios, oligohydramnios, patent 
foramen ovale, and complications of prematurity were not considered as associated abnormalities. Cryptorchidism and PDAs were included as additional abnormalities if the baby was term. Prematurity was defined as a delivery before 37 weeks gestation. Miscarriage (spontaneous termination) was a pregnancy ending spontaneously at $<20$ weeks gestation. The study included probands with gastroschisis and omphalocele (syndromic, nonsyndromic, and isolated) and excluded prune belly syndrome, limb-body wall complex, and umbilical hernias.

Gastroschisis and omphalocele were confirmed, along with associated anomalies, through physical examination by one of the authors (B.G.K.) in the pediatric cases. For prenatal cases, defects were described through targeted level II sonograms, which have a $<5 \%$ false-positive rate; autopsy/pathological data provided additional information for pregnancies ending in stillbirth, miscarriage, or elective termination; physical examination (B.G.K.) confirmed the diagnosis in newborns if the pediatrician or obstetrician requested a genetics consultation. Gastroschisis was defined as an anterior abdominal wall defect adjacent to the umbilicus with no sac or membrane. Omphalocele was defined as a midline anterior abdominal wall defect covered by the peritoneum and amniotic membrane. Cases that provided an inadequate or ambiguous description of the defect were excluded from the study. Cause of death was determined from the official cause of death listed on the autopsy report and its description of the hospital course and autopsy findings to support the listed cause of death. Environmental exposures were determined through an interview with one of the authors (B.G.K.) at the time of birth for pediatric cases and at the time of referral after the abnormal ultrasound in prenatal cases. Information about the general Tampa Bay population was obtained for comparison from the Department of Vital Statistics and Health Education.

The study was approved by the Institutional Review Board.

\section{RESULTS}

There were 127 cases of omphalocele ( 74 prenatal, 53 pediatric; Table 1). Of the 74 prenatally detected cases, 29 were liveborn and then referred to the USF Genetics Division, and 23 delivered elsewhere and outcome was unknown. Twelve pregnancies were electively terminated. Ten pregnancies ended in miscarriage; of those, five fetuses showed intrauterine growth retardation and other abnormalities, three had trisomy 18 , one had trisomy 21 , and one had an isolated omphalocele.

During this same period, there were 121 cases of gastroschisis (92 prenatal, 29 pediatric; Table 1). Of the two stillborn cases, one stillborn fetus had multiple anomalies, including hydrops, microtia, transposition of the great vessels, horseshoe kidney, 2-vessel cord, and bilateral syndactyly of fingers; the fetus was delivered intact. Chromosomal abnormality was suspected, but a karyotype was not performed. The other stillbirth had an isolated gastroschisis.

Probands with omphaloceles had a higher incidence of chromosome anomalies and other abnormalities. Of the 93
Table 1

Breakdown of 248 patients with gastroschisis or omphalocele in the Tampa Bay area from 1982 to 2000

\begin{tabular}{lr}
\hline Omphalocele $(N=127)$ & \\
Prenatal cases $(N=74)$ & 29 \\
Livebirths & 12 \\
Elective termination & 10 \\
Spontaneous termination & 23 \\
Delivered elsewhere & 0 \\
Stillbirths & \\
Pediatric cases $(N=52)$ & 50 \\
Gastroschisis $(N=121)$ & 5 \\
Prenatal cases $(N=92)$ & 2 \\
Livebirths & 33 \\
Elective termination & 2 \\
Spontaneous termination & \\
Delivered elsewhere & \\
Stillbirths & \\
Pediatric cases $(N=29)$ &
\end{tabular}

cases that had chromosome studies, 19 had an abnormal karyotype (20\%) (Table 2). In contrast, none of the 37 karyotyped probands with gastroschisis had abnormal results $(P=$ 0.007).

Ninety-three of 127 omphalocele cases had additional birth defects (73\%) (Table 3). Thirty-two of the 50 syndromic omphalocele were liveborn. The most common associated abnormality in the nonsyndromic was cardiac defects $(24 \%)$. Cases of cardiac defects included 16 probands with ventricular septal defect (VSD), 4 with atrial septal defect (ASD), 4 with tetralogy of Fallot, 2 with PDA, 2 with dextrocardia, 1 with ASD and VSD, and 1 with coarctation of the aorta, ASD, and VSD. Cases of umbilical cord malformation included 7 probands with a single umbilical artery, 2 with short cords, and 1 with an enlarged cord.

Table 2

Abnormal results in 93 karyotyped omphalocele probands

\begin{tabular}{lcc}
\hline \multicolumn{1}{c}{ Total abnormal } & $\mathrm{n}$ & $\%$ \\
\hline Trisomy 18 & 19 & $20 \%$ \\
Trisomy 21 & 11 & $12 \%$ \\
Trisomy 13 & 2 & $2 \%$ \\
Monosomy X & 1 & $1 \%$ \\
$46, X X, \operatorname{del}(18)(\mathrm{p} 11) / 46, X X, \mathrm{i}(18 \mathrm{q})$ & 1 & $1 \%$ \\
$46, \mathrm{XY}, \operatorname{inv}(16)(\mathrm{p} 11.1 \mathrm{q} 11.2)$ & 1 & $1 \%$ \\
$46, \mathrm{XX}, \operatorname{inv}(2)(\mathrm{p} 11 \mathrm{q} 12) \mathrm{mat}$ & 1 & $1 \%$ \\
$46, \mathrm{XY}, \operatorname{inv}(3)(\mathrm{p} 13 \mathrm{q} 11)$ & 1 & $1 \%$ \\
\hline
\end{tabular}


Table 3

Associated abnormalities in 93 probands with omphalocele and 29 probands with gastroschisis

\begin{tabular}{|c|c|c|}
\hline & $\mathrm{n}$ & $\%$ \\
\hline \multicolumn{3}{|l|}{ Omphalocele } \\
\hline Total & 93 & 73 \\
\hline Syndromic & 50 & 39 \\
\hline Chromosomal & 19 & 20 \\
\hline Beckwith-Wiedemann & 15 & 12 \\
\hline Pentalogy of Cantrell & 11 & 9 \\
\hline OEIS & 5 & 4 \\
\hline Nonsyndromic $^{a}$ & 43 & 34 \\
\hline Cardiac & 30 & 24 \\
\hline Cranial & 14 & 11 \\
\hline Urogenital & 13 & 10 \\
\hline Limb & 13 & 10 \\
\hline Umbilical & 10 & 8 \\
\hline Vertebral & 6 & 5 \\
\hline Bladder/cloacal exstrophy & 5 & 4 \\
\hline Neural tube & 4 & 3 \\
\hline Imperforate anus & 3 & 2 \\
\hline Diaphragmatic hernia & 3 & 2 \\
\hline Cleft lip/palate & 2 & 2 \\
\hline Meckel diverticulum & 2 & 2 \\
\hline Polydactyly & 1 & 1 \\
\hline \multicolumn{3}{|l|}{ Gastroschisis } \\
\hline Total & 29 & 23 \\
\hline Syndromic & 0 & 0 \\
\hline Nonsyndromic ${ }^{b}$ & 34 & 28 \\
\hline Cardiac & 14 & 12 \\
\hline Urogenital & 9 & 7 \\
\hline Limb & 3 & 2 \\
\hline Vertebral & 2 & 2 \\
\hline Neural tube & 2 & 2 \\
\hline Umbilical & 2 & 2 \\
\hline Cleft lip and palate & 1 & 1 \\
\hline
\end{tabular}

Value for Total, $P<0.001$.

${ }^{a}$ Cryptorchidism occurred in 9/38 liveborn males (24\%).

${ }^{b}$ Crytoorchidism occurred in 6/35 liveborn males (17\%).

In contrast, 29 of the 121 gastroschisis probands had additional anomalies $(23 \%)(P<0.001$; Table 3$)$. Cases of cardiac defects included 8 probands with VSD, 2 with ASD, 2 with tetralogy of Fallot, 1 with PDA, and 1 with transposition of the great vessels and VSD. Cases of umbilical cord malformation included 2 probands with a single umbilical artery. The two cases of neural tube defects were spina bifida aperta; both were among the pediatric cases. Information was not available as to whether the neural tube defects were surgically corrected. The single case of cleft lip and palate was a pregnancy electively terminated at 19 weeks and the information was gathered through the pathologist's autopsy report.

Among the 82 liveborn infants with omphalocele, the prematurity rate was $34 / 82(42 \%)$; for the 79 liveborn infants with gastroschisis, the prematurity rate was $45 / 79(57 \%)(P=0.18)$.

The mortality rate within the first year of life for the omphalocele group was 18/82 (22\%). The causes of death were not directly related to the omphalocele ( 9 respiratory distress, 4 congenital heart defects, 3 neural tube defects, 2 postoperative complications). One infant had a ruptured omphalocele but died of respiratory distress related to prematurity. In the gastroschisis group, the infant mortality rate was $7 / 79$ (9\%) $(P<$ 0.05 ). The causes of death in the infants with gastroschisis were 4 cases of congenital heart defects, 2 cases of postoperative complications, and 1 case of respiratory distress secondary to prematurity.

Four cases of gastroschisis were undiagnosed prenatally; of those, one case was undiagnosed due to lack of prenatal care. One omphalocele case was undiagnosed prenatally due to lack of prenatal care. Five cases of omphalocele were misdiagnosed on fetal sonogram as gastroschisis. Conversely, there were two cases of gastroschisis misdiagnosed as omphalocele on fetal sonogram.

Sixty-six of the 127 mothers of omphalocele probands (52\%) reported taking medication during the gestation, whereas 60 of 121 probands with gastroschisis (50\%) were exposed to medications during gestation (Table 4).

In the omphalocele group, drug abuse was reported in 6 of the 127 mothers (5\%); there were 2 cases of cocaine use, 1 cocaine and heroin, 1 THC and marijuana, 1 amphetamines, and 1 unspecified. In the gastroschisis group, 5 of 121 mothers (4\%) took street drugs during pregnancy $(P=1.0)$; there were 2 cases of cocaine and marijuana use, 1 marijuana, and 2 unspecified.

In the omphalocele group, 30 mothers smoked during pregnancy compared to 29 in the gastroschisis group ( $24 \%$ each) $(P$ $=0.88)$. Twenty-two $(17 \%)$ mothers with omphalocele probands drank alcohol during pregnancy, compared to 17 (14\%) in the gastroschisis group $(P=0.70)$. However, none of the mothers met the criteria for chronic alcoholism and none of their offspring showed evidence of fetal alcohol syndrome. Prenatal x-ray exposure was 8/127 (6\%) for omphalocele, and $4 / 121(3 \%)$ for gastroschisis $(P=0.30)$. There were 3 cases of gestational diabetes in the each group (3\%), reflecting the incidence of the general Tampa Bay area population.

There were two familial cases of omphalocele (1.6\%); the relationships were mother and full sister, respectively. Three probands with gastroschisis had a family history of the defect (2.4\%). The relationships to the probands were dizygotic twin sister, full sibling, and first cousin once removed, respectively.

Nine of the omphalocele probands were twins $(7.1 \%)$, whereas the incidence of twins is $1.1 \%$ in the general Tampa Bay area population $(P=0.001)$. There were 8 dizygotic pairs 
Table 4

Medication use during pregnancy in mothers of 127 probands with omphalocele and 121 probands with gastroschisis

Omphalocele, 66 cases (52\%)

Prenatal vitamins

OTC $^{a}$ analgesic

Antibiotic

OTC $^{a}$ cold medication

Corticosteroids

Iron

Synthroid

Anticonvulsant

Antidepressant

Birth control pills

Insulin

Gastroschisis, 60 cases (50\%)

Prenatal vitamins

$\mathrm{OTC}^{a}$ analgesic

Antibiotic

$\mathrm{OTC}^{a}$ cold medication

Corticosteroids

Iron

Insulin

$P=0.86$.

${ }^{a}$ Over-the-counter.

and 1 pair of monozygotic girls. All 9 pairs were discordant for the defect. In the monozygotic pair, twin A had the omphalocele as an isolated anomaly, whereas twin B had defects (exstrophy of the bladder, imperforate anus, and spinal abnormalities) that complemented her sister's omphalocele in the OEIS complex. Twin B died at age 9 hours due to respiratory arrest.

There were three dizygotic twin pregnancies in the gastroschisis group (2.5\%), with 1 pair concordant for the defect. A fourth pregnancy ended in miscarriage and a resorbed twin was suspected. The $\mathrm{DZ}$ twin prevalence was higher compared to the incidence in the general Tampa Bay area population $(P$ $=0.98)$. No consanguinity was reported in either group.

Mean maternal age was 21.8 years for gastroschisis and 27.2 years for omphalocele. Maternal age distribution for all cases of gastroschisis and omphalocele, in comparison to the general Tampa Bay area population, is shown in Fig. 1. Racial composition was similar to that of the Tampa Bay area population (Caucasian 75\%, African American 13\%, Hispanics 10\%, Oriental 2\%). For the omphalocele group, 93/127 (73\%) were Caucasian, 20/127 (16\%) African American, 11/127 (9\%) Hispanic, and 4/127 (3\%) Asian. In the gastroschisis group, 93/121 (77\%) were Caucasian, 14/121 (12\%) African American, and 13/121 (11\%) Hispanic.

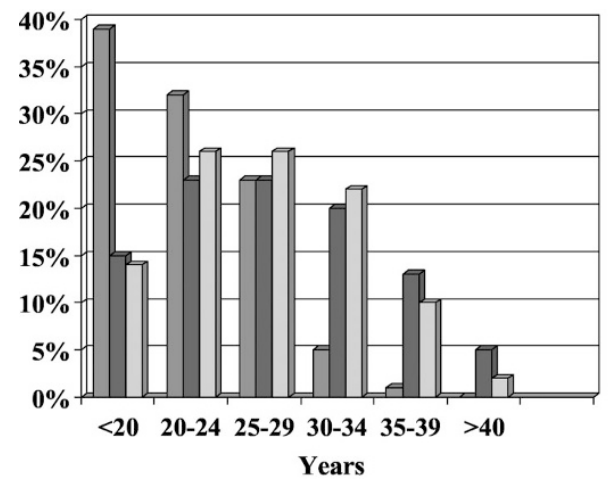

$\square$ Gastroschisis $\square$ Omphalocele $\square$ Gen. Population

Fig. 1. Characteristics of study participants as compared to general Tampa Bay area population.

\section{DISCUSSION}

This study was initiated because an increase in the number of gastroschisis cases was observed by the Genetics Division at USF. In this study, the ratio of omphalocele to gastroschisis was 127 to 121 or 1:1, compared to the expected ratio of 3:2. If only the liveborn were included, the ratio remains 1:1, with 82 cases of omphalocele and 79 cases of gastroschisis. Other studies around the world have found increases in gastroschisis prevalence, along with a decrease in omphalocele..$^{4,6-9}$ Rankin et al. ${ }^{7}$ reported an increase in the incidence of gastroschisis without a corresponding change in omphalocele. Theories to account for these incidence changes have included an environmental agent, inaccurate classification, limited family histories, and a higher familial recurrence risk. ${ }^{10}$ No cases of gastroschisis were recorded in British Columbia before 1969 because the condition was unknown and previous cases were diagnosed as omphalocele. ${ }^{11}$

There were no reported cases of omphalocele stillbirths, although the figure in the literature is $11 \%$ to $12 \%(P<0.001) .{ }^{9}$ However, excluding elective terminations and pregnancies of unknown outcome, the spontaneous termination or miscarriage rate was $25 \%$ (10/39). Perhaps differences in criteria for stillbirth versus miscarriage contribute to the discrepancy, or maybe there was a bias in reporting.

The percentage of associated anomalies was 73\% for omphaloceles versus $23 \%$ for gastroschisis. In addition, the proportion of cases with chromosomal abnormalities was also higher in the omphalocele group (20\%) compared to the gastroschisis group (0\%). Similar findings have been reported in previous studies, $5,7,9$ with cardiac defects as the most common associated anomaly with both omphalocele and gastroschisis. ${ }^{5}$

The prematurity rate in the two groups was higher than the $15 \%$ rate in the general Tampa Bay area population, although the difference between the two groups is not significant. Reports of prematurity rates in gastroschisis have been historically higher, ranging from $40 \%$ to $67 \%,{ }^{6}$ compared to the $10 \%$ to $23 \%$ rate for omphalocele. ${ }^{5}$ Perhaps differences in prematurity criteria may account for the higher prematurity rate in this omphalocele group compared to previous studies.

The 1-year mortality rate for the omphalocele group was significantly higher than the gastroschisis group. The 4 cases of 
congenital heart defects in the gastroschisis group were 2 probands with tetralogy of Fallot, 1 with VSD, and 1 with transposition of the great vessels with VSD; none of the children had additional gastrointestinal defects.

Cocaine and ephedrine have long been suspects as teratogens for gastroschisis. These chemicals are vasoconstrictors, potentially causing occlusion of fetal abdominal wall arteries, with the defect resulting from inadequate blood supply. ${ }^{12}$ In this series, only two mothers admitted use of cocaine during pregnancy. Cold medications were taken frequently and the majority of mothers did not remember which ones they used. Many of the over-the-counter cold medications contain ephedrine. However, similar findings were noted in the omphalocele group. Thus, ephedrine-containing drugs and cocaine did not appear to play a role in the abdominal wall defects.

The recurrence risk for nonsyndromic abdominal wall defects has been postulated to be low $(<1 \%),{ }^{10,13,14}$ but Torfs and Curry ${ }^{10}$ found a $3.5 \%$ recurrence risk for gastroschisis by extending the pedigree to second cousins. They suggested that incomplete family histories result in underreported familial cases ${ }^{10}$ and that counseling of families with a case of gastroschisis should change accordingly. Also, inaccurate diagnosis or documentation in medical records may change the frequency of recurrence of ventral wall defects within a family..$^{10,13}$ Theories on mode of inheritance have ranged from Mendelian monogenic to multifactorial. ${ }^{10,13-15}$

In this study, there was a significant increase of twin pairs only among the omphalocele probands. It is known that twins have a higher rate of birth defects. ${ }^{4}$ Monozygotic (MZ) twins are at a higher risk than dizygotic (DZ) twins. ${ }^{4}$ In this series all twin pairs but one in the gastroschisis group were discordant for the defect. In the concordant pair, one of the twins had a congenital heart defect in addition to the gastroschisis. The mother drank two beers and smoked 1 to 3 cigarettes a day. Smoking and alcohol have been associated with gastroschisis. ${ }^{16}$

In this study, the gastroschisis group had younger mothers than did the omphalocele group and the general population. Although mothers under 20 years of age comprise $14 \%$ of the general population, they comprised $39 \%$ of the gastroschisis group. There were no mothers 40 years or older in the gastroschisis group. In an earlier study, ${ }^{17}$ the mean maternal age was 20.4 years for gastroschisis, as compared to 28.0 years for the general population. Boyd et al. ${ }^{18}$ also found younger mothers in their gastroschisis group. Torfs et al. ${ }^{19}$ found the incidence of gastroschisis to be highest among mothers under 20 years old, with the incidence declining steeply with increasing maternal age; only $7 \%$ of mothers were over 29 years of age in their group. The reason for the younger maternal age is unknown. ${ }^{19}$

There is no guarantee that every patient with gastroschisis or omphalocele was referred to practice, such as those whose mothers had no appreciable prenatal risk factors or family history. Gastroschisis patients are subject to being underreferred because of the low rate of associated abnormalities. For unknown reasons, stillborn omphalocele patients were also un- derrepresented in this study. In addition, chart review studies are also vulnerable to interpretive bias in obtaining data, but this bias was limited through physical examination of liveborn children by the same physicians, and reviewing official sonogram and autopsy reports.

The 248 patients with gastroschisis and omphalocele evaluated through the USF genetics clinics (pediatric and prenatal) corroborated the reported observation in other medical facilities/specialties that since the 1960s, gastroschisis is increasing in prevalence relative to omphalocele, ${ }^{4,6-9}$ and may be more common than isolated omphaloceles in liveborns. Although there is no clear reason for the change in numbers, this statistic should be reflected in the textbooks that still present omphalocele as more common.

\section{ACKNOWLEDGMENT}

This study was funded in part by a Children Medical Service grant from Florida Health and Rehabilitative Services.

\section{References}

1. Mayer T, Black R, Matlak M, Johnson D. Gastroschisis and omphalocele: An eightyear review. Ann Surg 1980;192:783-787.

2. Moore TC, Stokes GE. Gastroschisis: Report of two cases treated by a modification of the gross operation for omphalocele. Surgery 1953;33:112-120.

3. Duhamel B. Embryology of exomphalos and allied malformations. Arch Dis Child 1963;38:142-147.

4. Buyse ML, editor. Birth defects encyclopedia. Cambridge: Blackwell Scientific Publications; 1990:1289-1290.

5. Stevenson RE, Hall JG, Goodman RM. Omphalocele and gastroschisis. In: Stevenson RE, Hall JG, Goodman RM, editors. Human malformations and related anomalies. Oxford: Oxford University Press; 1993:879-882.

6. Suita S, Okamatsu T, Yamamoto T. Changing profile of abdominal wall defects in Japan: results of a national survey. J Pediatr Surg 2000;35:66-71.

7. Rankin J, Dillon E, Wright C. Congenital anterior abdominal wall defects in the north of England, 1986-1996: occurrence and outcome. Prenat Diagn 1999;19:662668.

8. Kitchanan S, Patole SK, Muller R, Whitehall JS. Neonatal outcome of gastroschisis and exomphalos: A 10-year review. J Pediatr Child Health 2000;36:428-430.

9. Tan KH, Kilby MD, Whittle MJ, Beattie BR, Booth IW, Botting BJ. Congenital anterior abdominal wall defects in England and Wales 1987-93: Retrospective analysis of OPCS data. BMJ 1996;313:903-906.

10. Torfs CP, Curry CJ. Familial cases of gastroschisis in a population-based registry. Am J Med Genet 1993;45:465-467.

11. Baird PA, MacDonald EC. An epidemiologic study of congenital malformations of the anterior abdominal wall in more than half a million consecutive live births. Am J Hum Genet 1981;33:470-478.

12. Werler M, Sheehan JE, Mitchell AA. Association of vasoconstrictive exposure with risks of gastroschisis and small intestinal atresia. Epidemiol 2003;14:349-354.

13. Bugge M, Petersen MB, Christensen MF. Monozygotic twins discordant for gastroschisis: Case report and review of the literature of twins and familial occurrence of gastroschisis. Am J Med Genet 1994;52:223-226.

14. Yang P, Beaty TH, Khoury MJ, Chee E, Stewart W, Gordis L. Genetic-epidemilogic study of omphalocele and gastroschisis: Evidence for heterogeneity. Am J Med Genet 1992;44:668-675.

15. Pryde PG, Greb A, Isada NB, Johnson MB, Klein M, Evans MI. Familial omphalocele: consideration in genetic counseling. Am J Med Genet 1992;44:624-627.

16. Torfs CP, Velie EM, Oechsli FW. A population-based study of gastroschisis: demographic, pregnancy, and lifestyle risk factors. Teratology 1994;50:44-53.

17. Penman DG, Fisher RM, Noblett HR, Soothill PW. Increase in incidence of gastroschisis in the south west of England in 1995. Br J Obstet Gynec 1998;105:328-331.

18. Boyd PA, Bhattacharjee A, Gould S, Manning N, Chamberlain P. Outcome of prenatally diagnosed anterior abdominal wall defects. Arch Dis Child Fetal Neonatal Ed 1998;78:F209-F213.

19. Torfs C, Curry C, Roeper P. Gastroschisis. J Pediatr 1990;116:1-6. 